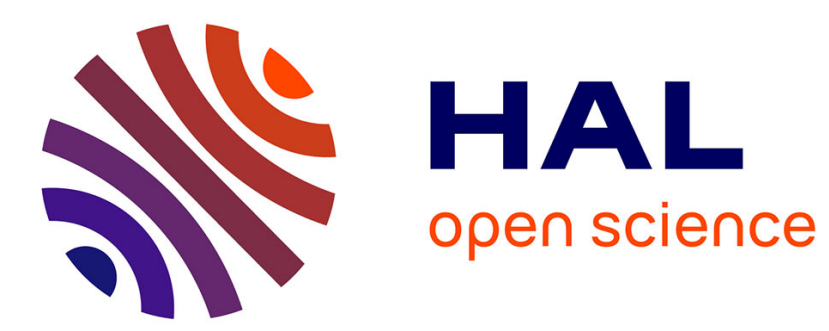

\title{
Évolution sémantique des prépositions spatiales de l'ancien au moyen français
}

Benjamin Fagard

\section{To cite this version:}

Benjamin Fagard. Évolution sémantique des prépositions spatiales de l'ancien au moyen français. Lingvisticae Investigationes, 2002, 25 (2), pp.311-338. halshs-00320036

\section{HAL Id: halshs-00320036 \\ https://shs.hal.science/halshs-00320036}

Submitted on 11 Dec 2015

HAL is a multi-disciplinary open access archive for the deposit and dissemination of scientific research documents, whether they are published or not. The documents may come from teaching and research institutions in France or abroad, or from public or private research centers.
L'archive ouverte pluridisciplinaire HAL, est destinée au dépôt et à la diffusion de documents scientifiques de niveau recherche, publiés ou non, émanant des établissements d'enseignement et de recherche français ou étrangers, des laboratoires publics ou privés. 


\title{
Évolution sémantique des prépositions spatiales de l'ancien au moyen français
}

\author{
Benjamin Fagard
}

\section{Présentation}

\subsection{Introduction}

Le but de cette étude est de mettre au jour la présence éventuelle de régularités dans l'évolution sémantique, en analysant un groupe de prépositions (vers, envers, devers, pardevers) sur une période s'étalant de l'ancien au moyen français (11e-16e siècles), à partir d'un corpus de textes informatisés. La question des constantes de l'évolution sémantique a été remise à l'honneur ces dernières années par les études sur la grammaticalisation, dans le cadre de l'analyse des changements de sens subis par un mot lors de son passage du lexique à la grammaire. On trouve à ce propos deux approches opposées, consistant à considérer pour l'une que l'espace est primaire et à l'origine de toute évolution sémantique, pour l'autre que l'espace peut servir de base sémantique mais que ce n'est là qu'une tendance parmi d'autres, et que l'espace n'a pas de statut privilégié en tant que domaine sémantique. Nous avons cherché à trouver dans l'évolution sémantique de vers, envers, devers et pardevers des indices nous permettant d'avoir une meilleure idée concernant la validité de ces deux approches pour les prépositions ${ }^{1}$.

Après une brève introduction à ces deux approches (1.2.), nous exposerons notre méthode (1.3.), en expliquant nos choix de catégorisation sémantique, puis notre corpus (1.4.). Nous présenterons ensuite nos résultats (2.1. à 2.4.), en indiquant pour chaque préposition le nombre d'occurrences par siècle, et la fréquence de chaque type d'emploi (spatial, relationnel, temporel, comparatif). Nous donnerons, également, pour chaque préposition, une liste de ses différents emplois, suivie d'une explication de son sémantisme et de son évolution. Nous discuterons enfin l'ensemble des résultats, en proposant une analyse synthétique de l'évolution de vers, envers, devers et pardevers (3.).

\footnotetext{
${ }^{1}$ Sur lesquelles je fais actuellement une thèse.
} 


\subsection{Espace et évolution sémantique}

À l'origine de l'hypothèse de la primarité du spatial est l'idée que l'espace est premier du point de vue cognitif : après L. Talmy (1996, 2000), on trouve cette idée par exemple chez B. Kryk-Kastovsky (1996 : 329) citant S. Levinson : " on retrouve la pensée 'spatiale' dans presque tous les autres domaines de pensée » (spatial thinking intrudes into thinking about almost all other domains), ou chez C. Zelinsky-Wibbelt (1996 : 528), selon qui « l'espace est le principe d'organisation majeur dans tous les domaines cognitifs " (Space is the major organizational principle in all cognitive domains). Enfin, selon R. Dirven (1993 : 73), « les conceptualisations spatiales de base peuvent être projetées sur un 'espace mental', et le sont effectivement, c'est-àdire qu'elles peuvent former des chaînes de sens couvrant divers domaines conceptuels tels que le temps, l'état, l'aire, la manière ou le moyen, la circonstance, la cause ou la raison, etc. " (The basic spatial conceptualizations can be and are projected onto 'mental space', i.e., they may form chains of meaning covering various conceptual domains such as time, state, area, manner or means, circumstance, cause or reason, etc.). Appliquant cette idée aux prépositions, il évoque « les extensions de sens d'une préposition, partant de l'espace physique et passant par le temps pour arriver à des domaines plus abstraits » (1993 : 76) (The extensions of the meanings of a preposition from physical space via time into more abstract domains), et C. Zelinsky-Wibbelt parle des prépositions comme «étant à l’origine des prédicats spatiaux» (1993:10) (Prepositions, being originally spatial predications). On retrouve la même idée chez $\mathrm{R}$. Langacker qui dit des prépositions qu'elles désignent des relations spatiales, " étendues métaphoriquement à de nombreux autres domaines cognitifs » (1993 : 287) (Extended metaphorically to many other cognitive domains). On pourrait donc établir pour les prépositions des chaînes sémantiques comme celle que proposent E. Traugott et E. König: Espace > Temps > Cause > Concession (1991 : 189-218).

Cependant, l'espace n'est pas toujours vu comme universellement premier. B. Heine, U. Claudi \& F. Hünnemeyer ont ainsi montré l'existence de chaînes sémantiques du type Personne / partie du corps > Objet $>$ Activité $>$ Espace $>$ Temps $>$ Qualité (1991 : 32). La même chose a été démontrée à propos des prépositions par S. Svorou (1994) et M. Haspelmath (1997 : 140). Ce dernier explique d’abord que « la grande majorité des langues emploie une adposition (ou un cas) originellement spatiale (spatial) pour signifier une relation temporelle (The large majority of languages employ an originally spatial adposition (or case) to signal a temporal relationship), mais montre ensuite qu'il y a des exceptions dans de nombreuses langues, et que ce n'est pas un phénomène universel. Jackendoff (1990) va plus loin en affirmant que «autant la structure spatiale que la structure temporelle sont des 
projections d'une organisation abstraite qui peut être appliquée, avec une spécialisation suffisante, à n'importe quel domaine » (Both spatial structure and temporal structure are instantiations of an abstract organization that can be applied with suitable specialization to any field ; cité par M. Haspelmath).

\subsection{Méthode : typologie des emplois}

Considérant que le sens d'une préposition dépend en partie du contexte, nous avons prêté une attention particulière à ce dernier pour établir les différents emplois de chaque préposition. En adoptant la terminologie de C. Vandeloise (1986), nous parlerons ici de site (landmark pour S. Svorou (1994 :9), après Langacker) et de cible (trajector) pour désigner respectivement le régime de la préposition (site) et ce qui régit la préposition (cible). Ainsi, dans la phrase Vait s'en Brandan vers le grant mer (exemple 1), la cible est Brandan, c'est l'entité à localiser (la préposition ayant ici un emploi spatial) et le site est la mer, qui permet la localisation. Nous montrerons ici, en faisant la liste des différents emplois des prépositions étudiées, quels traits définitoires nous avons employés pour définir chacun d'eux.

\subsubsection{Emplois spatiaux}

Pour ces emplois, la préposition sert à désigner une relation purement spatiale entre deux entités, comme le but, la situation, la provenance. On trouve des emplois spatiaux du type but (exemple 1), où la cible est en mouvement en direction du site. Le plus souvent, la cible est humaine, et le site peut être humain mais est en général un lieu. Dans notre corpus, ce type d'emploi se trouve dans $70 \%$ des cas avec les verbes suivants: aller, venir, retourner, approcher, traire, adresser, chevaucher, poindre, soit des verbes de mouvement.

(1) Vait s'en Brandan vers le grant mer / U sout par Deu que dout entrer Le Voyage de Saint Brendan (1112), v.157

(Brendan s'en va vers la haute mer, où il savait par Dieu qu'il devait entrer)

On trouve également des emplois spatiaux de type statique (exemple 2), où la situation décrite est une orientation de la cible par rapport au site, ou bien un changement de posture, un geste de la cible en direction du site, la direction du regard, ce que L. Talmy appelle «mouvement fictif» (fictive motion, Talmy 1996 : 211). Cible et site peuvent être humains ou non humains. Ce type d'emploi se trouve le plus souvent avec les verbes suivants : tourner (tourner son visage vers / se tourner), regarder, 
tendre (la main, un objet), être (au sens de "se trouver ", " être situé »), s'incliner, mais également comme complément du nom pour permettre la détermination ou la localisation d'un objet ou d'une personne, sans verbe : la fenêtre vers le jardin.

(2) Guardez amunt devers les porz d'Espaigne : / Veeir poez, dolente est la rereguarde

La chanson de Roland (1080), v.1103

(Regardez en haut, vers les cols d'Espagne: vous pouvez le voir, l'arrière-garde est en mauvaise posture)

Enfin, on trouve moins fréquemment des emplois spatiaux de type provenance (exemple 3), qui sont le pendant des emplois de but : la cible s'éloigne du site, pour se rapprocher du locuteur. Cet emploi ne se trouve quasiment qu'avec les verbes venir et revenir.

(3) Demantres est l'ancre rompue / par coi la nes s'estoit tenue; / devers la terre vint li vanz, / a la mer bota la nef anz

Enéas (1155), v.5791

(Pendant ce temps, l'ancre qui retenait le navire s'est rompue; le vent vint de la terre, et poussa le navire à la mer)

\subsubsection{Emplois relationnels}

Pour ces emplois, la préposition sert à indiquer une relation entre deux êtres humains, et en particulier à exprimer les sentiments, l'attitude de l'un pour l'autre. Cible et site sont donc toujours (ou presque) humains, et toujours agents d'un verbe d'action, de comportement, de sentiment, la cible étant sujet de ce verbe (premier actant), et le site le destinataire ou le partenaire (pour l'emploi de type neutre) de cette action.

On trouve d'abord un emploi de type neutre (exemple 4), où il s'agit de situations d'interaction sans intention positive ou négative de la cible pour le site. Cible et site peuvent même, à la différence du détrimentaire et du positif (cf. infra), se trouver sur le même plan, avec des verbes comme être en paix et avoir trêve. Cet emploi se rencontre d'ailleurs le plus souvent (60\% des cas) avec les verbes être (en secret, attentif, de la partie de, en serment, en merci, ...) et avoir (trêve...), ainsi qu'avec le verbe faire (également dans diverses constructions...).

(4) Si doi frere, Jehans et Terris, tretiierent viers ceuls de Cambrai pour ravoir le corps

Chroniques de Froissart (1369-1377), p. 350

(Ses deux frères, Jean et Terris, traitèrent avec ceux de Cambrai pour récupérer le corps) 
On trouve également un emploi de type détrimentaire (exemple 5), où il y a une intention ou une action de la cible visant à nuire au site. Les verbes avoir (courroux, haine, défense, force) et être (courroucé, cruel, fel, fier) sont les plus fréquents (de 25 à $40 \%$ des occurrences selon les époques), mais la part d'autres verbes comme oeuvrer (avec félonie / cruellement, malement), méprendre, défendre ou se défendre, faire (couardise, déloyauté) n’est pas négligeable.

(5) Signe qu'il n'est point entaché de ce fol vice d'orgueil qui procure hayne envers toutes personnes

Mémoires de Commynes (1490-1505), p. 66

(Signe qu’il n’est point entaché du vice démesuré qu’est l’orgueil, qui procure haine envers toutes et tous)

On trouve enfin un emploi de type bénéfactif (exemple 6), où il s'agit d'une interaction "positive » entre êtres humains, ou plutôt d'une action, d'un sentiment ou d'une attitude bénéfique d'une personne pour une autre. On trouve, pour cet emploi, en majorité (60\% des occurrences) les verbes avoir (amour, amitié, bonne pensée, courage, désir, envie) et être (amical, loyal).

(6) Monseigneur, qui trescourtois et gracieux estoit, mesmement tousjours vers les dames, luy dist : "M'amye, pource que vous estes belle fille et bonne, et que j'ayme bien vostre mary, il me prend pitié et compassion de vostre fait

Les cent nouvelles nouvelles (1456-1467), p. 39

(Monseigneur, qui était très courtois et gracieux, surtout et toujours envers les dames, lui dit: "Mon amie, parce que vous êtes belle et bonne, et que j’apprécie votre mari, j’ai pitié et compassion pour ce qui vous arrive »)

\subsubsection{Emploi temporel}

L'emploi temporel (exemple 7) est assez rare avec les prépositions étudiées ici. Il permet de donner une approximation de la date, de l'heure, du moment auquel s'effectue une action, ou qui constitue la limite temporelle de cette action; le sujet de l'action est en général humain, et le site est invariablement un élément temporel : heure, moment de la journée, durée.

(7) Et Renart, devers meriane, / por reposer se traist ariere / en mi le fonz de sa taisniere

Le roman de Renart, Branche I (1180-1200), v.502 
(Et Renart, vers midi, se retira vers le fond de sa tanière pour se reposer)

\subsubsection{Emploi comparatif}

Avec l'emploi comparatif, les prépositions vers et envers mettent en regard l'ensemble d'une classe avec un élément défini tiré de cette classe, afin de mettre en valeur son aspect exceptionnel, comme dans l'exemple 8. On peut donc le trouver avec des constructions diverses, mais surtout avec le verbe être attribuant à la cible la qualité qui lui permet de prendre cet aspect « exceptionnel ».

(8) Theüter ot Povrepeleux, / qui mout fu bien chevalerex; / n'i ot cheval tant amast chier, / vers lui sont li autre eschacier

Le roman de Thèbes (1150-1155), v.6298

(Theüter avait Povrepeleux, qui était très courageux; il l'aimait plus qu’aucun autre cheval : les autres ne sont qu’échassiers comparés à lui)

\subsection{Corpus}

Nous avons choisi de découper en «tranches chronologiques » notre corpus, qui regroupe une cinquantaine de textes littéraires du 11e au 16e. Nous l'avons établi à partir d'un corpus informatisé mis au point par l'UMR 8503, la Base de Français Médiéval, exploitable en ligne grâce à un logiciel développé par Serge Heiden (Weblex). Afin d'obtenir une vision diachronique des phénomènes étudiés, nous avons constitué six groupes de textes, contenant respectivement les textes du 11e (groupe A), du 12e (groupe B), du 13e (groupe C), du 14e (groupe D), du 15e (groupe E) et du 16e (groupe F) siècles ${ }^{2}$.

Nous nous fierons plus particulièrement aux résultats obtenus pour les groupes $\mathrm{B}$ à $\mathrm{E}$, étant donné que ce sont les parties du corpus les plus homogènes, contenant entre 400 000 et 800000 mots (les groupes A et F en contenant entre 40000 et 50 000).

\begin{tabular}{|c|c|c|c|}
\hline Siècle & nombre de mots & nombre de textes & groupe \\
\hline $11 e$ & 38423 & 4 & $\mathrm{~A}$ \\
\hline $12 e$ & 641044 & 19 & $\mathrm{~B}$ \\
\hline $13 e$ & 777067 & 13 & $\mathrm{C}$ \\
\hline $14 e$ & 389946 & 4 & $\mathrm{D}$ \\
\hline
\end{tabular}

\footnotetext{
${ }^{2}$ Le critère retenu pour le classement chronologique d'un texte est la date du premier manuscrit (sauf pour La vie de saint Louis de Jean de Joinville, dont les manuscrits s’étalent de 1298 à 1309).
} 


\begin{tabular}{|c|c|c|c|}
\hline $15 e$ & 591393 & 8 & $\mathrm{E}$ \\
\hline $16 e$ & 54426 & 1 & $\mathrm{~F}$ \\
\hline Total & 2492299 & 49 & $\mathrm{G}$ \\
\hline
\end{tabular}

\section{Résultats : évolution sémantique des prépositions vers, envers, devers, pardevers, du 11e au 16e siècle.}

\subsection{Vers}

Nous présentons ici les résultats obtenus pour les graphies vers, viers, ver.

\subsubsection{Tableaux}

a) occurrences ${ }^{3}$

\begin{tabular}{|l|l|c|c|c|c|c|c|}
\hline \multicolumn{2}{|l|}{ Siècle } & $11 e$ & $12 e$ & $13 e$ & $14 e$ & $15 e$ & $16 e$ \\
\hline \multirow{4}{*}{ Corpus } & Nombre de textes & 4 & 19 & 13 & 4 & 8 & 1 \\
\hline \multirow{3}{*}{ Spatial } & Nombre de mots & 40000 & 640000 & 780000 & 390000 & 590000 & 50000 \\
\hline & But & 7 & 305 & 258 & 248 & 228 & 14 \\
\cline { 2 - 8 } & Statique & 8 & 114 & 76 & 25 & 43 & 5 \\
\cline { 2 - 8 } & Provenance & & & & & & \\
\hline \multirow{3}{*}{ Relationnel } & Neutre & 4 & 103 & 74 & 11 & 14 & \\
\cline { 2 - 8 } & Détrimentaire & 1 & 249 & 132 & 16 & 11 & \\
\cline { 2 - 8 } & Bénéfactif & & 45 & 27 & 6 & 6 & \\
\hline \multicolumn{2}{|l|}{ Temporel } & & 9 & 15 & 5 & 4 & 1 \\
\hline \multicolumn{2}{l|l}{ Comparatif } & & 4 & 2 & & 2 & 1 \\
\hline \multicolumn{2}{l|}{ Total des occurrences } & 20 & 829 & 584 & 311 & 308 & 21 \\
\hline
\end{tabular}

b) pourcentages ${ }^{4}$

\begin{tabular}{|l|c|c|c|c|c|c|c|}
\hline \multicolumn{2}{|l|}{ Siècle } & $11 e$ & $12 e$ & $13 e$ & $14 e$ & $15 e$ & $16 e$ \\
\hline
\end{tabular}

\footnotetext{
${ }^{3}$ Ce tableau donne, comme les suivants (2.2.1.a, 2.3.1.a, 2.4.1.a), le nombre d’occurrences présentes dans chaque groupe de textes (siècle par siècle, du $11^{\mathrm{e}}$ au $16^{\mathrm{e}}$ ) pour chaque type d'emploi. Nous rappelons de plus dans les premières lignes du tableau le nombre de textes et le nombre de mots (approximation à la dizaine de mille) correspondant à chaque partie du corpus.

${ }^{4}$ Ce tableau, comme les suivants (2.2.1.b, 2.3.1.b, 2.4.1.b), indique la part de chaque type de sens, pour un siècle donné, par rapport à l'ensemble des occurrences (par exemple, au $11^{\mathrm{e}}$ siècle, les occurrences spatiales de but représentent pour notre corpus 35 \% des occurrences totales pour ce siècle).
} 


\begin{tabular}{|l|l|c|c|c|c|c|c|}
\hline \multirow{2}{*}{ Spatial } & Statique & 40 & 13,75 & 13,01 & 8,04 & 13,96 & 23,8 \\
\cline { 2 - 8 } & Provenance & & & & & & \\
\hline \multirow{4}{*}{ Relationnel } & Neutre & 20 & 12,42 & 12,67 & 3,54 & 4,55 & \\
\cline { 2 - 8 } & Détrimentaire & 5 & 30,04 & 22,61 & 5,14 & 3,57 & \\
\cline { 2 - 8 } & Bénéfactif & & 5,43 & 4,62 & 1,93 & 1,95 & \\
\hline \multirow{2}{*}{ Temporel } & & 1,09 & 2,57 & 1,61 & 1,3 & \\
\hline Comparatif & & 0,48 & 0,34 & & 0,65 & 4,76 \\
\hline \multicolumn{2}{l|l}{ Total } & $100 \%$ & $100 \%$ & $100 \%$ & $100 \%$ & $100 \%$ & $100 \%$ \\
\hline
\end{tabular}

Soit, pour la proportion spatial / relationnel / autre :

\begin{tabular}{|l|c|c|c|c|c|c|}
\hline Siècle & $11 e$ & $12 e$ & $13 e$ & $14 e$ & $15 e$ & $16 e$ \\
\hline Spatial & 75 & 50,54 & 57,19 & 87,78 & 87,99 & 90,48 \\
\hline Relationnel & 25 & 47,89 & 39,9 & 10,61 & 10,07 & 0 \\
\hline Autre & 0 & 1,57 & 2,91 & 1,61 & 1,95 & 9,52 \\
\hline Total & $100 \%$ & $100 \%$ & $100 \%$ & $100 \%$ & $100 \%$ & $100 \%$ \\
\hline
\end{tabular}

\subsubsection{Sens observés}

a) sens spatial / but :

(9) Vait s'en Brandan vers le grant mer / U sout par Deu que dout entrer Le Voyage de Saint Brendan (1112), v.157

(Brendan s'en va vers la haute mer, où il savait par Dieu qu'il devait entrer)

b) sens spatial / statique :

(10) Et quant monseignor Ancelin les vit et en fu saisis, si leva ses mains vers le chiel et rendi graces a Dieu; et fu le plus lié homme dou monde, pour ce que adonc fu certains que il raveroit son frere de la prison ou il estoit Chronique de Morée (1320-1324), p. 379

(il leva ses mains vers le ciel et rendit grâces à Dieu)

c) sens relationnel / neutre :

(11) Si doi frere, Jehans et Terris, tretiierent viers ceuls de Cambrai pour ravoir le corps

Chroniques de Froissart (1369-1377), p. 350 
(Ses deux frères, Jean et Terris, traitèrent avec ceux de Cambrai pour récupérer le corps)

d) sens relationnel / détrimentaire :

(12) De ce, fet ele, n'ai ge cure, / car vers l'autre estes tuit parjure, / qui jurastes par anz l'annor, / li baron et li vavasor Le roman de Thèbes (1150-1155), v.7406 (De cela, fit-elle, je n'ai cure, car vous êtes tous parjures envers l'autre, vous barons et vavasseurs qui lui avez prêté serment auparavant)

e) sens relationnel / bénéfactif :

(13) Monseigneur, qui trescourtois et gracieux estoit, mesmement tousjours vers les dames, luy dist: "M'amye, pource que vous estes belle fille et bonne, et que j'ayme bien vostre mary, il me prend pitié et compassion de vostre fait »

Les cent nouvelles nouvelles (1456-1467), p. 39

(Monseigneur, qui était très courtois et gracieux, surtout et toujours envers les dames, lui dit: "Mon amie, parce que vous êtes belle et bonne, et que j'apprécie votre mari, j'ai pitié et compassion pour ce qui vous arrive »)

f) sens temporel :

(14) Vers mie nuit tuit s'aclasserent, / et cil qui sus el chastel erent, / se gaitent bien, ne dorment pas

Enéas (1155), v.4903

(Vers minuit, tous s'assoupirent, tandis que ceux qui sont au-dessus, dans le château, font bien le guet, et ne dorment pas)

g) sens comparatif :

(15) Theüter ot Povrepeleux, / qui mout fu bien chevalerex; / n'i ot cheval tant amast chier, / vers lui sont li autre eschacier

Le roman de Thèbes (1150-1155), v.6298

(Theüter avait Povrepeleux, qui était très courageux; il l'aimait plus qu'aucun autre cheval : les autres ne sont qu'échassiers comparés à lui) 


\subsubsection{Sémantisme et évolution}

La préposition vers est polysémique : on en trouve 7 types d'emplois différents dans notre corpus (spatiaux, relationnels, temporels et comparatifs). Elle est aussi très fréquente, bien plus que ses « composés » devers, pardevers et envers.

L'emploi le plus prototypique de vers est, dès les premiers textes, le sens spatial de but; ce sens apparaît d'ailleurs avant les autres si l'on se fie d'une part à sa présence dans la Passion de Saint Léger (vers 1000), d'autre part aux dictionnaires, qui datent les autres sens de vers du début du 12e siècle. Le caractère prototypique de l'emploi spatial de but ne fait que croître par la suite, en particulier entre le 13e et le 14 e siècles, avec une très légère baisse au 15e et au 16e siècles.

On peut noter d'autre part que les sens relationnels forment une part non négligeable des emplois de vers en ancien français (du 11e au 13e siècle) mais baissent brutalement entre le $13 \mathrm{e}$ et le $14 \mathrm{e}$ siècle, pour se maintenir à un niveau très bas par la suite.

\subsection{Devers}

Nous présentons ici les résultats obtenus pour les graphies devers, de-vers, deviers, de vers. $^{5}$

\subsubsection{Tableaux}

a) occurrences

\begin{tabular}{|c|c|c|c|c|c|c|c|}
\hline \multicolumn{2}{|l|}{ Siècle } & $11 e$ & $12 e$ & $13 e$ & $14 e$ & $15 e$ & $16 e$ \\
\hline \multirow[b]{2}{*}{ Corpus } & Nombre de textes & 4 & 19 & 13 & 4 & 8 & 1 \\
\hline & Nombre de mots & 40000 & 640000 & 780000 & 390000 & 590000 & 50000 \\
\hline \multirow{4}{*}{ Spatial } & But & 2 & 12 & 8 & 172 & 290 & 1 \\
\hline & Statique & 4 & 55 & 24 & 55 & 37 & \\
\hline & Provenance & 5 & 17 & 18 & 7 & & \\
\hline & Neutre & & 8 & 13 & 47 & 9 & \\
\hline
\end{tabular}

\footnotetext{
${ }^{5}$ Nous avons en effet choisi de traiter sur le même plan les graphies devers et de vers, même s'il n'est pas sûr qu'il s'agisse toujours de la même forme. Nous l'avons fait pour deux raisons : d'une part, les graphies de vers sont extrêmement minoritaires, trop pour être étudiées indépendamment; d'autre part, rien ne dit qu'une graphie devers dans une édition ne correspond pas à un de vers sur le manuscrit, ou à une abréviation, sans compter les graphies de-vers : nous laissons donc de côté le problème pour cette étude, sans oublier que c'en est un (le F.E.W. (von Wartburg, 1958-1968) explique d'ailleurs qu'il est très délicat de faire la distinction entre ces deux constructions).
} 


\begin{tabular}{|l|l|l|c|c|c|c|c|}
\cline { 2 - 7 } & Détrimentaire & & 5 & 0 & 4 & 1 & \\
\cline { 2 - 8 } Relationnel & Bénéfactif & & 3 & 2 & 1 & 1 & \\
\hline Comparatif & & & & & & \\
\hline Temporel & & 1 & 0 & 0 & 6 & \\
\hline Total des occurrences & 11 & 101 & 65 & 286 & 344 & 1 \\
\hline
\end{tabular}

b) pourcentages

\begin{tabular}{|l|l|c|c|c|c|c|c|}
\hline \multicolumn{2}{|l|}{ Siècle } & $11 \mathrm{e}$ & $12 \mathrm{e}$ & $13 \mathrm{e}$ & $14 \mathrm{e}$ & $15 \mathrm{e}$ & $16 \mathrm{e}$ \\
\hline \multirow{4}{*}{ Spatial } & But & 18,18 & 11,88 & 12,31 & 60,14 & 84,3 & 100 \\
\cline { 2 - 8 } & Statique & 36,36 & 54,46 & 36,92 & 19,23 & 10,76 & \\
\cline { 2 - 8 } & Provenance & 45,45 & 16,83 & 27,69 & & 2,45 & \\
\hline \multirow{3}{*}{ Relationnel } & Neutre & & 7,92 & 20 & 16,43 & 2,62 & \\
\cline { 2 - 8 } & Détrimentaire & & 4,95 & & 1,4 & 0,29 & \\
\cline { 2 - 8 } & Bénéfactif & & 2,97 & 3,08 & 0,35 & 0,29 & \\
\hline \multicolumn{2}{l|}{ Comparatif } & & & & & & \\
\hline \multicolumn{2}{l|}{ Temporel } & & 0,99 & & & 1,74 & \\
\hline \multicolumn{2}{l|}{ Total } & $100 \%$ & $100 \%$ & $100 \%$ & $100 \%$ & $100 \%$ & $100 \%$ \\
\hline
\end{tabular}

Soit, pour la proportion spatial / relationnel / autre :

\begin{tabular}{|l|c|c|c|c|c|c|}
\hline Siècle & $11 e$ & $12 e$ & $13 e$ & $14 e$ & $15 e$ & $16 e$ \\
\hline Spatial & 100 & 83,17 & 76,92 & 81,82 & 95,06 & 100 \\
\hline Relationnel & & 15,84 & 23,08 & 18,18 & 3,2 & \\
\hline Autre & & 0,99 & & & 1,74 & \\
\hline Total & $100 \%$ & $100 \%$ & $100 \%$ & $100 \%$ & $100 \%$ & $100 \%$ \\
\hline
\end{tabular}

\subsubsection{Sens observés}

a) sens spatial / but

(16) Messires Lois d'Espagne, qant il fu descendus et desarmés, il se traist devers la tente de mesire Carle de Blois

Chroniques de Froissart (1369-1377), p. 517

(Monseigneur Louis d'Espagne, quand il fut descendu et se fut débarrassé de ses armes, alla vers la tente de Monseigneur Charle de Blois) 
b) sens spatial / statique

(17) Guardez amunt devers les porz d'Espaigne : / Veeir poez, dolente est la rereguarde

La chanson de Roland (1080), v.1103

(Regardez en haut, vers les cols d'Espagne: vous pouvez le voir, l'arrière-garde est en mauvaise posture)

c) sens spatial / provenance

(18) Demantres est l'ancre rompue / par coi la nes s'estoit tenue; / devers la terre vint li vanz, / a la mer bota la nef anz

Enéas (1155), v.5791

(Pendant ce temps, l'ancre qui retenait le navire s'est rompue; le vent vint de la terre, et poussa le navire à la mer)

d) sens relationnel / neutre

(19) É si feinst qu'il haïst e mal feïst á la gent de Israel pur çó que meillur grace truvast devers le rei

Les quatre livres des rois (1150-1190), p. 52

(Et ainsi il feint de haïr les gens d'Israël, et de leur vouloir du mal, pour trouver meilleure grâce auprès du roi)

e) sens relationnel / détrimentaire

(20) Pour ce temps, avoit li contes de Hainnau une haine couverte moult grande deviers son serourge, le roi Phelippe de France; je vous dirai quelle et pourquoi

Chroniques de Froissart (1369-1377), p. 255

(En ce temps-là, le comte de Hainaut nourrissait en secret une très grande haine, contre son beau-frère, le roi de France Philippe; je vous dirai sa nature et son origine)

f) sens relationnel / bénéfactif

(21) Madame, qui de tout ce fut bien aise et voist que sa gracieuseté devers les maistres ouvriers lui a aidié, aussi sa largesse bien employé, dist en riant a ses femmes [...] 
Jehan de Saintré (1456), p. 57

(Madame, qui fut bien contente de tout cela et vit que son amabilité envers les contremaîtres l'avait aidée, et que sa générosité était bien employée, dit en riant à ses femmes [...])

g) sens temporel

(22) Et Renart, devers meriane, / por reposer se traist ariere / en mi le fonz de sa taisniere

Le roman de Renart, Branche I (1180-1200), v.502

(Et Renart, vers midi, se retira vers le fond de sa tanière pour se reposer)

\subsubsection{Sémantisme et évolution}

La préposition devers est polysémique : elle a, comme vers, des emplois spatiaux, relationnels, ainsi qu'un sens temporel dont les dictionnaires consultés ne font pas mention. Elle est prototypiquement spatiale, plus encore que vers, et présente trois emplois spatiaux distincts : but, statique, provenance. ${ }^{6}$ Au sein des emplois spatiaux, il n'y a pas d'emploi prototypique «stable » : c'est successivement la provenance au 11e siècle, puis le statique aux 12e et 13e siècles, et le but ensuite. Le seul emploi relationnel qui se trouve assez fréquemment est le neutre (en particulier dans des constructions du type traiter devers, s'acquitter devers).

On peut signaler le fait que les emplois de devers se démarquent de ceux de vers du 11e au 13e siècles, pour deux raisons : d'une part, chacune connaît un emploi que l'autre n'a pas - c'est la provenance pour devers (ce qui s'explique par sa combinaison avec de), le comparatif pour vers; on pourrait ajouter que les emplois bénéfactif et détrimentaire sont exceptionnels pour devers mais pas pour vers. D'autre part, les emplois spatiaux sont bien plus prototypiques pour devers que pour vers. Mais ce qui est remarquable est que les deux prépositions se rejoignent au 14e siècle : vers a plus d'emplois spatiaux, devers plus d'emplois relationnels. Et la fréquence de devers augmente au point de rejoindre celle de vers.

Ainsi, les prépositions vers et devers sont partiellement synonymes dès l'ancien français, comme en atteste l'exemple 23, où ces prépositions sont utilisées en coordination (avec un emploi spatial); mais elles deviennent plus proches encore sémantiquement en moyen français (exemples 24-25). On voit bien cette proximité sémantique dans les exemples suivants :

au 13e :

\footnotetext{
${ }^{6}$ Il est clair que cet emploi peut correspondre à une construction de type [de [vers $\left.\left.Y\right]_{S P}\right]_{S P}$ avec des syntagmes prépositionnels enchâssés (où la séquence de vers n'est pas grammaticalisée), ou bien à une construction [devers $Y]_{S P}$ avec un seul morphème prépositionnel et un syntagme prépositionnel simple.
} 
(23) Bruians point desous devers destre, / Et li rois Baudins vers senestre Le bel inconnu (avant 1214), v.5702

(Bruians charge dessous, vers la droite, et le roi Baudins vers la gauche)

au 14e :

(24) Qant mesires Hervis de Lion et ses gens veirent venir l'esfort, si se retraissent fort viers la chité et deviers la porte, et cachierent lor proie dedens

Chroniques de Froissart (1369-1377), p. 497

(Quand monseigneur Hervis de Lyon et ses gens virent venir la troupe, ils se retirèrent rapidement vers la cité et vers la porte, et cachèrent leur butin à l'intérieur)

au 15e :

(25) Ledit prince de Salerne alla à Venise, pour ce qu'il y avoit grant accointance, et avecques luy trois de ses nepveux, filz du prince de Besygnan, où demandèrent conseil, comme m'a dit ledit prince, à la Seigneurie, où il leur plaisoit myeulx qu'ilz tirassent : ou vers ledit duc de Lorreyne ou devers le roy de France ou d'Espaigne?

Mémoires de Commynes (1490-1505), p. 10

(Ledit prince de Salerne alla à Venise, où il connaissait beaucoup de monde, et emmena trois de ses neveux, fils du prince de Besygnan; ils y demandèrent conseil, comme m'a dit ce prince, à la Seigneurie, pour savoir où il valait mieux qu'ils aillent : auprès dudit duc de Lorraine ou auprès du roi de France ou d’Espagne ?)

\subsection{Pardevers}

Nous présentons ici les résultats obtenus pour les graphies pardevers et par devers.

\subsubsection{Tableaux}

a) occurrences 


\begin{tabular}{|c|c|c|c|c|c|c|c|}
\hline \multirow[b]{2}{*}{ Corpus } & Nombre de textes & 4 & 19 & 13 & 4 & 8 & 1 \\
\hline & Nombre de mots & 40000 & 640000 & 780000 & 390000 & 590000 & 50000 \\
\hline \multirow{3}{*}{ Spatial } & But & & 3 & 4 & 13 & 11 & \\
\hline & Statique & & 3 & 26 & 12 & 1 & \\
\hline & Provenance & & & & & & \\
\hline \multirow{3}{*}{ Relationne } & Neutre & & & & & & \\
\hline & Détrimentaire & & & & & & \\
\hline & Bénéfactif & & & 13 & 3 & 2 & \\
\hline \multicolumn{8}{|l|}{ Comparatif } \\
\hline \multicolumn{2}{|l|}{ Temporel } & & & & & & \\
\hline \multicolumn{2}{|c|}{ Total des occurrences } & 0 & 6 & 43 & 31 & 14 & 0 \\
\hline
\end{tabular}

b) pourcentages

\begin{tabular}{|c|c|c|c|c|c|c|c|}
\hline \multicolumn{2}{|l|}{ Siècle } & $11 e$ & $12 e$ & $13 e$ & $14 e$ & $15 e$ & $16 e$ \\
\hline \multirow[b]{3}{*}{ Spatial } & But & & 50 & 9,3 & 41,94 & 78,57 & \\
\hline & Statique & & 50 & 60,47 & 48,39 & 7,14 & \\
\hline & Provenance & & & & & & \\
\hline \multirow[b]{3}{*}{ Relationne } & Neutre & & & 30,23 & 9,68 & 14,29 & \\
\hline & Détrimentaire & & & & & & \\
\hline & Bénéfactif & & & & & & \\
\hline \multicolumn{2}{|l|}{ Comparatif } & & & & & & \\
\hline \multicolumn{2}{|l|}{ Temporel } & & & & & & \\
\hline \multicolumn{2}{|l|}{ Total } & & $100 \%$ & $100 \%$ & $100 \%$ & $100 \%$ & \\
\hline
\end{tabular}

Soit, pour la proportion spatial / relationnel :

\begin{tabular}{|l|c|c|c|c|c|c|}
\hline Siècle & $11 e$ & $12 e$ & $13 e$ & $14 e$ & $15 e$ & $16 e$ \\
\hline Spatial & & 100 & 69,77 & 90,32 & 85,71 & \\
\hline Relationnel & & & 30,23 & 9,68 & 14,29 & \\
\hline Autre & & & & & & \\
\hline Total & & $100 \%$ & $100 \%$ & $100 \%$ & $100 \%$ & \\
\hline
\end{tabular}

\subsubsection{Sens observés}

a) sens spatial / but :

(26) Car, après les graces a Dieu de par elle et sa fille rendues, se mettent a 
chemin par devers l'ermitage, ou l'ermite leur vient au devant, qui de Dieu les salue et beneist

Les cent nouvelles nouvelles (1456-1467), p. 102

(Car, une fois qu'elle et sa fille eurent rendu grâces à Dieu, elles se mettent en chemin vers l'hermitage, où l'hermite vient au-devant d'elles, les saluant et les bénissant au nom de Dieu)

b) sens spatial / statique :

(27) Ovec li, au mien essient, / a bien .xxx. serganz a conte; / et l'autre porte garde Honte, / qui ovre par devers midi

Le Roman de la Rose de Guillaume de Lorris (1225-1230), v.3857 (Avec lui, à mon avis, il faut bien compter trente hommes d'armes; et Honte garde l'autre porte, qui s'ouvre vers le midi)

c) sens relationnel / neutre :

(28) Qui a pluseurs resons, soit par devers le defendeur ou par devers le demandeur, il doit dire toutes ses resons qu'il aime le meins avant et les meilleurs au derrain

Coutumes de Beauvaisis (1283), p. 132

(Celui qui a plusieurs choses à dire, par-devers le défendant ou pardevers le plaignant, doit dire celles qu'il aime le moins avant, et les meilleures en dernier)

\subsubsection{Sémantisme et évolution}

La préposition pardevers est la seule de notre corpus à être peu polysémique. Elle n’apparaît que tardivement (à la fin du 12e siècle selon les dictionnaires consultés, mais au milieu d'après notre corpus), est peu fréquente, et ne présente que des emplois spatiaux de but et statique, qui sont prototypiques, et des emplois relationnels neutres. Il n’y a pas d'emploi temporel, conformément à ce que disent les dictionnaires consultés.

Comment se fait-il alors que pardevers se soit maintenu jusqu'en français moderne avec un sémantisme restreint et une fréquence très faible, alors que devers, au sémantisme plus large et à la fréquence plus grande, n’est plus employé dès le dixseptième siècle ? Il semble que ce soit lié au fait que, dès le départ et probablement en raison de sa composition (avec comme préfixe la préposition par-), elle présente une spécificité sémantique qu’on peut voir dans l’exemple suivant : 
aprochier son hoost ne la ville de Calais, fors que par une voie laquelle venoit tout droit le grant cemin, ou par les dunes de la mer, ou par deviers Ghines, Melq et Oie, ou il avoit grant fuisson de fosses et de lieus imposibles pour si grande hoost a passer

Chroniques de Froissart (1369-1377), p. 826

(Ce roi s'imaginait que les Français ne pouvaient arriver jusqu'à lui, ni se rapprocher de son armée ou de la ville de Calais, sauf en passant tout droit par la grande route, ou par les dunes donnant sur la mer, ou par Ghines, Melq et Oie, où il y avait un grand nombre de fossés et de lieux impossibles à traverser pour une si grande armée)

On voit ici que ce qui distingue le régime de par devers des régimes observés pour les prépositions vers, envers et devers est que ce n'est pas un but ou une origine mais un lieu de passage, de la même manière que les régimes de par. La préposition pardevers s'utilise lorsqu'il s'agit de répondre à la question quā (par où ?).

\subsection{Envers}

Nous présentons ici les résultats obtenus pour les graphies envers, enviers, emvers, enver, ensvers.

\subsubsection{Tableaux}

a) occurrences

\begin{tabular}{|c|c|c|c|c|c|c|c|}
\hline \multicolumn{2}{|l|}{ Siècle } & $11 e$ & $12 e$ & $13 e$ & $14 e$ & $15 e$ & $16 e$ \\
\hline \multirow{3}{*}{ 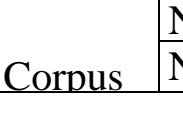 } & Nombre de textes & 4 & 19 & 13 & 4 & 8 & 1 \\
\hline & Nombre de mots & 40000 & 640000 & 780000 & 390000 & 590000 & 50000 \\
\hline & But & 4 & 12 & 5 & & 3 & \\
\hline \multirow[t]{2}{*}{ Spatial } & Statique & 3 & 5 & 4 & 1 & 5 & 1 \\
\hline & Provenance & & & & & & \\
\hline \multirow{3}{*}{ Relationnel } & Neutre & 2 & 25 & 27 & 11 & 29 & 13 \\
\hline & Détrimentaire & 1 & 38 & 30 & 5 & 21 & 6 \\
\hline & Bénéfactif & & 14 & 5 & 3 & 15 & 5 \\
\hline \multicolumn{2}{|l|}{ Comparatif } & & 7 & 4 & 1 & 3 & \\
\hline \multicolumn{2}{|l|}{ Temporel } & 2 & & & & & \\
\hline \multicolumn{2}{|c|}{ Total des occurrences } & 12 & 101 & 75 & 21 & 76 & 25 \\
\hline
\end{tabular}




\begin{tabular}{|l|l|c|c|c|c|c|c|}
\hline \multicolumn{2}{|l|}{ Siècle } & $11 e$ & $12 e$ & $13 e$ & $14 e$ & $15 e$ & $16 e$ \\
\hline \multirow{4}{*}{ Spatial } & But & 33,33 & 11,88 & 6,67 & & 3,95 & \\
\cline { 2 - 8 } & Statique & 25 & 4,95 & 5,33 & 4,76 & 6,58 & 4 \\
\cline { 2 - 8 } & Provenance & & & & & & \\
\hline \multirow{4}{*}{ Relationnel } & Neutre & 16,67 & 24,75 & 36 & 52,38 & 38,16 & 52 \\
\cline { 2 - 8 } & Détrimentaire & 8,33 & 37,62 & 40 & 23,81 & 27,63 & 24 \\
\cline { 2 - 8 } & Bénéfactif & & 13,86 & 6,67 & 14,29 & 19,74 & 20 \\
\hline Comparatif & & 6,93 & 5,33 & 4,76 & 3,95 & \\
\hline \multicolumn{2}{l|}{ Temporel } & 16,67 & & & & & \\
\hline \multicolumn{2}{l|}{ Total } & $100 \%$ & $100 \%$ & $100 \%$ & $100 \%$ & $100 \%$ & $100 \%$ \\
\hline
\end{tabular}

Soit, pour la répartition spatial / non spatial :

\begin{tabular}{|l|c|c|c|c|c|c|}
\hline Siècle & $11 e$ & $12 e$ & $13 e$ & $14 e$ & $15 e$ & $16 e$ \\
\hline Spatial & 58,33 & 16,83 & 12 & 4,76 & 10,53 & 4 \\
\hline Relationnel & 25 & 74,25 & 82,67 & 90,48 & 85,53 & 96 \\
\hline Autre & 16,67 & 8,91 & 5,33 & 4,76 & 3,95 & \\
\hline Total & $100 \%$ & $100 \%$ & $100 \%$ & $100 \%$ & $100 \%$ & $100 \%$ \\
\hline
\end{tabular}

\subsubsection{Sens observés :}

a) sens spatial / but :

(30) Mes de fain fu en grant destrece: / envers une vile s'adresce / en la maison d'un tainturier / qui mout savoit de ce mestier

Le roman de Renart, Branche I (1180-1200), v.2296

(mais la faim le tenaillait: il se dirige vers une ville, dans la maison d'un teinturier qui connaissait bien son métier)

b) sens spatial / statique :

(31) Carles guardat amunt envers le ciel, / Veit les tuneires e les venz e les giels / E les orez, les merveillus tempez, / $E$ fous e flambes $i$ est apareillez

La chanson de Roland (1080), v.2532

(Charles regarda en haut, vers le ciel, vit le tonnerre, le vent, le gel et les brises, les tempêtes formidables, le feu et les flammes prêts à tomber)

c) sens relationnel / neutre : 
(32) Li mieldre reis ot a nom Charlemaine; / Cil aleva volentiers dolce France; / Deus ne fist terre qui envers lui n'apende

Le couronnement de Louis (mi XIIe), v.16

(Le meilleur roi avait pour nom Charlemagne; celui-ci éleva volontiers la douce France; Dieu n’a fait de terre qui ne lui soit soumise)

d) sens relationnel / détrimentaire :

(33) Signe qu'il n'est point entaché de ce fol vice d'orgueil qui procure hayne envers toutes personnes

Mémoires de Commynes (1490-1505), p. 66

(signe qu’il n’est point entaché du vice démesuré qu’est l’orgueil, qui procure haine envers toutes et tous)

e) sens relationnel / bénéfactif :

(34) Affin que ceulx qui viendroyent après luy fussent ung peu plus pyteux envers le peuple et moins aspres à pugnir qu'il n'avoit esté

Mémoires de Commynes (1490-1505), p. 86

(afin que ceux qui lui succéderaient soient un peu plus miséricordieux envers le peule et moins âpres à punir qu’il n’avait été)

f) sens temporel :

(35) Envers lo vesprae envers lo ser / dunc lo revidren doi fidel Passion (1000), v.445

(Au moment des vêpres, vers le soir, deux fidèles le revirent donc)

g) sens comparatif :

(36) En li ot totes bones taches : / el ne fu oscure ne brune, / mes reluisant come la lune / envers qui les autres estoilles / resemblent petites chandailles

Le Roman de la Rose de Guillaume de Lorris (1225-1230), v.997

(Il y avait en elle toutes les qualités : elle n’était pas obscure ni sombre, mais étincelait comme la lune auprès de qui les autres étoiles ressemblent à de petites chandelles) 


\subsubsection{Sémantisme et évolution}

Comme vers et devers, la préposition envers est polysémique: on trouve dans les textes des emplois spatiaux, relationnels, temporels et comparatifs. Elle est cependant, seule de ce groupe de prépositions, prototypiquement relationnelle dès le 12e siècle, et ce jusqu'en français moderne où c'est son seul emploi. L'emploi le plus fréquent est le détrimentaire (12e-13e) puis le neutre (14e-16e), mais on trouve à toutes les époques à partir du 12e les trois types d'emplois relationnels.

Il y a une synonymie partielle entre vers et envers, comme en témoignent les quelques exemples de coordination (toujours pour des emplois relationnels) entre les deux prépositions, ou les cas d'emploi avec le même type de construction chez le même auteur :

(37) Car tant ai fait vers la reïne / culcher ne dei od la meschine / e envers la meschine tant fait / que ne puet mie estre retrait

Tristan de Thomas (1170-1175), v.449-452

(j'en ai tant fait vers la reine que je ne dois pas coucher avec cette jeune fille, et j'en ai tant fait envers cette demoiselle que je ne peux revenir en arrière)

(38) Oiez de qoi on vos apele : / Que Tristran n'ot vers vos amor / De puteé ne de folor / Fors cele que devoit porter / Envers son oncle et vers sa per

Tristan de Beroul (1180), v.4191-4195

(Écoutez ce qu'on vous dit: que Tristan n'eut pour vous d'amour de débauche ni de folle conduite, rien d'autre que l'amour qu'il devait porter à son oncle et à sa dame)

(39) Ou voit le roi, prinst l'en a apeller : / « Sire, dist il, vers moi en entendéz. / Jusqu'a set mois voil le jor respiter. » / Et dist li rois : "Volentiers et de gréz. " / Li gentiz hom nel mist en oublier. / Ou voit la dame, prinst l'an a apeller. / Dist li cuens : "Damme, envers moi entendéz, / Une parole voz voil dire et conter »

Ami et Amile (1200), v.811

(Il voit le roi et se met aussitôt à l’appeler : « Sire, dit-il, écoutez-moi. Je veux repousser le jour de sept mois », et le roi répond : «Volontiers, de bon gré ». Le gentilhomme ne l'oublia pas; dès qu'il vit la dame, il se mit à l'appeler. Le comte dit: «Dame, écoutez-moi, je veux vous raconter quelque chose »)

(40) Je ne sçauroye dire vers qui Nostre Seigneur s'est monstré plus 
courroucé : envers luy, qui mourut soudainement en ce champ, sans guères languir, ou vers ses subjectz?

Mémoires de Commynes (1490-1505), p. 58

(Je ne saurais dire envers qui Notre Seigneur s'est montré plus courroucé : envers lui, qui mourut soudainement en ce champ, sans agonie, ou envers ses sujets ?)

\section{Discussion}

Il faut distinguer deux périodes dans l'évolution sémantique de vers, envers et devers : 11e-13e et 14e-16e siècles, - la préposition pardevers ne présentant pas de réelle évolution sémantique décelable dans notre corpus. Pour la première période, on peut représenter le sémantisme de ces prépositions par un axe allant du relationnel au spatial, comme le montre le schéma 1 où devers et envers sont aux deux pôles, et vers entre le centre et le pôle spatial.



Ceci explique qu'on peut trouver vers et envers ou vers et devers en coordination, mais pas envers et devers. Il y a donc une certaine différenciation sémantique, mais qu'il ne faut pas prendre comme identique pendant toute cette période. D’abord parce qu'elle n'est pas tout à fait acquise au 11e, où on trouve les prépositions vers, envers et devers avec des emplois peu prototypiques, comme l'emploi temporel d'envers dans la Passion; en fait, les prépositions vers, envers et devers ont à ce moment-là indistinctement les emplois du latin adversus, avec en plus l'emploi temporel ${ }^{7}$. Ensuite parce qu'elle n'est que partielle, comme en attestent justement les exemples de coordination (23-25 et 37-40), ainsi que le fait que devers a des emplois relationnels dans 7 textes et envers des emplois spatiaux dans 10 textes aux 12e-13e siècles (sur 32 textes au total), tandis que vers apparaît avec des sens spatiaux et relationnels dans la quasi-totalité de ces textes. Enfin parce qu'elle s'accentue progressivement entre le 11e et le 13e : le schéma 1 n'est donc pas à prendre comme statique; en particulier, vers se rapproche petit à petit de devers, ce qui explique peutêtre que cette dernière préposition perde progressivement en fréquence face à la proximité sémantique grandissante d'une préposition plus polyvalente (puisque les

\footnotetext{
${ }^{7}$ F. Gaffiot (1934) donne pour adversus préposition : «1) en face de, en se dirigeant vers, contre; 2) (fig.) contre, à l'encontre de; 3) vis-à-vis de, à l'égard de, en s'adressant à; 4) en comparaison de (rare) ».
} 
emplois non spatiaux de devers sont exceptionnels) et surtout beaucoup plus fréquente qu'elle.

Pour la deuxième période, la représentation serait plutôt celle du schéma 2 , où vers et devers sont sur le même plan.

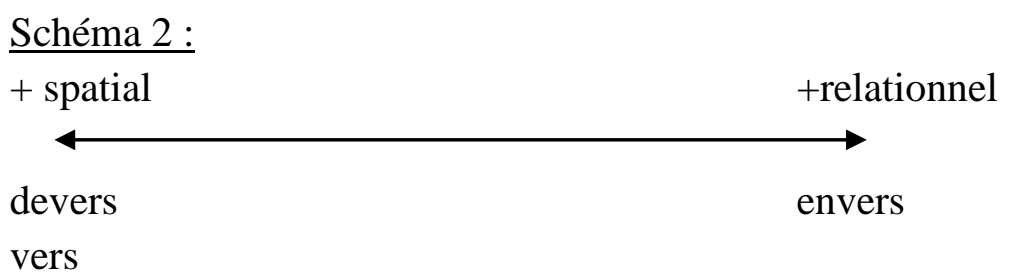

On a en effet pour cette période une quasi-synonymie de vers et devers, liée à l'évolution sémantique importante de vers entre le 13e et le 14e siècles (cf. 2.1.3.). On s'attendrait à ce que cela entraîne la disparition de devers, d'autant plus que sa fréquence avait baissé sans cesse du 11e au 13e. C'est l'inverse qui se produit: les deux prépositions ont aux 14e et 15e siècles non seulement le même type d'emplois, mais une fréquence identique.

Comment l'expliquer ? Si la fréquence plus grande de vers dans la première période est due à une plus grande généralité sémantique, cela ne peut plus jouer dans la deuxième période, puisqu'il y a quasi-équivalence des deux prépositions au niveau sémantique (mis à part les emplois comparatifs, soit moins de $1 \%$ des occurrences de vers). Rien ne privilégie plus l'emploi de vers, ce qui explique que la préposition devers soit choisie par les locuteurs environ une fois sur deux.

Au 16e siècle, cependant, notre corpus ne donne quasiment aucun emploi de devers : l'emploi qu'en fait (ou plutôt que n'en fait pas) Rabelais est-il représentatif de son siècle, et les locuteurs du 16e ont-ils fini par choisir entre les deux, éliminant un devers trop semblable à vers ? En tout état de cause, devers disparaît du français vers le milieu du 17e siècle, si l'on se fie aux données de Frantext, tandis que la préposition vers continue à être employée jusqu'en français moderne, avec ses emplois spatiaux et temporels.

Face à ce tandem vers / devers, la préposition envers se cantonne de plus en plus dans des emplois relationnels; elle n'est pas en concurrence avec les autres prépositions, vers et devers, et peut donc se maintenir avec ces emplois jusqu'en français moderne.

\section{Conclusion}

Nous avons montré dans cette étude que les prépositions vers, envers, devers et 
pardevers ne permettent pas de voir une quelconque évolution sémantique partant du spatial, puisque tous les emplois attestés pour ces prépositions sont déjà présents dès les premiers textes, et même dès le latin classique.

Elles constituent cependant un exemple intéressant d'évolution sémantique par les jeux de répartition des sens et de spécialisation que l'on peut observer de l'ancien au moyen français : on passe d'un état de langue où les prépositions vers, envers et devers sont plus ou moins indifférenciées (11e siècle) à un état de langue où ces prépositions se polarisent progressivement en spatiale (devers), relationnelle (envers) et spatiale/relationnelle (vers) (12e-13e siècles), puis à un état de langue où la polarisation est presque complète entre deux prépositions spatiales en concurrence (vers et devers) et une préposition relationnelle (envers) (14e-16e siècles).

C'est de ce dernier état de langue que vient la situation de ces prépositions en français moderne: la spécialisation sémantique de chacune ayant atteint sa limite, elles ne peuvent plus du tout entrer dans les mêmes constructions (vers et envers s'excluent mutuellement), et la concurrence entre vers et devers a entraîné la disparition de cette dernière au 17e siècle. La préposition pardevers semble rester en marge de cette opposition sémantique, avec des sens propres qui se précisent peu à peu et resteront jusqu'en français moderne.

Il resterait à voir quand s'est réellement amorcé le déclin de devers, et à quel point elle est synonyme de vers aux 16e et 17e siècles : apparaissent-elles dans les mêmes contextes ou sont-elles en distribution complémentaire ? Peut-il encore y avoir en français classique des emplois avec coordination de vers et envers, ce qui est possible au moins jusqu'au 15e siècle et semble impensable en français moderne?

\section{Corpus de textes}

\section{A : textes du 11e}

Cultura e lingua francese delle origini nella 'Passion' di Clermont-Ferrand., éd. D’Arco Silvio Avalle, 1962, Milan / Naples : R. Ricciardi. Date : vers 1000.

Saint Léger., éd. D’Arco Silvio Avalle, 1967. In Studia Ghisleriana (titre original : Sant Lethgier (X secolo). Nuova edizione critica con una nota introduttiva), II, vol. 3, Pavia. 349-362. Date : vers 1000.

La vie de saint Alexis. éd. Christopher Storey, 1968, Genève : Droz. Date : milieu 11e. La chanson de Roland, éd. Gérard Moignet, 1970, Paris : Bordas. Date : 1080.

\section{$B$ : textes du $12 e$}

Benedeit : Le voyage de saint Brendan, éd. I. Short et B. Merrilees, 1979, Anglo-Norman Text Society. Date : 1112. 
Gormont et Isembart, éd. Alphonse Bayot, 1931 (3e édition revue), Paris : Champion. Date : 1130.

Le roman de Thèbes, éd. Guy Raynaud de Lage, 1966-1968, Paris : Champion. Date : 1150-1155.

Les quatre livres des rois, éd. Ernst Robert Curtius, 1911, Dresden : Gesellschaft für Romanische Litteratur (Max Niemeyer). Date : 1150 / vers 1190.

Le couronnement de Louis, éd. E. Langlois, 1925, 2e édition revue, Paris : Champion. Date : mi 12e.

Enéas, éd. J.J. Salverda de Grave, 1973 et 1983, 2 vol., Paris : Champion. Date : 1155. Marie de France, Les Lais, éd. J. Rychner, 1966, Paris : Droz. Date : 1160.

Chrétien de Troyes, Erec et Enide, éd. Mario Roques, 1952, Paris : Champion. Date : 1164.

Lai de Narcisse, éd. M. Thiry-Stassin et M. Tyssens, 1976, Paris : Les Belles Lettres. Date : 1165-1175.

Thomas, Tristan, éd. Félix Lecoy, 1991, Paris : Champion. Date : 1170-1175. Chrétien de Troyes, Cligès, éd. A. Micha, 1957, Paris : Champion. Date : 1176.

Gautier d'Arras, Eracle, éd. Guy Raynaud de Lage, 1976, Paris : Champion. Date : 11761184.

Chrétien de Troyes, Le chevalier de la charette, éd. Mario Roques, d’après la copie Guiot, 1958, Paris : Champion. Date : 1177-1181.

Chrétien de Troyes, Yvain, le chevalier au lion, éd. K. Uitti, 1990-92, Paris : Bibliothèque de la Pléiade. Date : vers 1177-1181.

Conon de Béthune, Les Chansons de Conon de Béthune, éd. Axel Wallensköld, 1968 [1921], Paris : Champion. Date : 1180-1190.

Beroul, Tristan, éd. L.M. Defourques, 1947, Paris : Champion. Date : 1180.

Le roman de Renart, Branche I, éd. Mario Roques, d’après le manuscrit de Cangé, 1948, Paris : Champion. Date : 1180-1200.

Chrétien de Troyes, Perceval ou le Conte du Graal, éd. Félix Lecoy, 1973-75, Paris : Champion. Date : 1181-1185.

\section{$C:$ textes du $13 e$}

Le roman de Renart, Branches X-XI, éd. Mario Roques, d’après le manuscrit de Cangé, 1958, Paris : Champion. Date : début 13e.

Robert de Clari, La conquête de Constantinople, éd. Philippe Lauer, 1924, Paris : Champion. Date : début 13e.

Ami et Amile, éd. P.F. Dembowski, 1969, Paris : Champion. Date : 1200. Jean Renart, L'Escoufle, éd. F. Sweetser, 1974, Genève : Droz. Date : 1200.

Jean Renart, Le roman de la rose ou de Guillaume de Dole, éd. Félix Lecoy, 1962, Paris : Champion. Date : 1210.

Renaut de Beaujeu, Le Bel Inconnu, éd. G. Perrie Williams, 1967, Paris : Champion (2 édition). Date : avant 1214. 
Gautier de Coinci, Les Miracles de Nostre Dame, éd. V. Frederic Koenig, 1961 et 1966, Genève : Droz. Date : 1218-1227.

La quête du saint Graal, éd. A. Pauphilet, 1923, Paris : Champion. Date : 1220.

Guillaume de Lorris, Le roman de la rose, éd. Félix Lecoy, 1965, Paris : Champion. Date : 1225-1230.

La mort du roi Arthur, éd. Jean Frappier, 1954, Genève : Droz. Date : 1230.

Tristan en prose, éd. Philippe Ménard, 1987, Genève : Droz. Date : fin 13e.

La châtelaine de Vergy, éd. Guy Raynaud de Lage et L. Foulet, 1921, Paris : Champion. Date : milieu 13e.

L'Atre Périlleux, roman de la table ronde, éd. Brian Woledge, 1936, Champion. Date : avant 1268.

Jean de Meun, Le roman de la rose, éd. Félix Lecoy, 1965, Paris : Champion. Date : 12691278.

Philippe de Beaumanoir, Coutumes de Beauvaisis, éd. A. Salmon, 1970 [1900], Paris. Picard. Date : 1283 (première rédaction).

\section{$D$ : textes du 14e}

Jean de Joinville, La vie de saint Louis, éd. J. Monfrin, 1996, Paris. Garnier Flammarion. Date : 1298-1309.

Chronique de Morée, éd. J. Longnon, 1911, Paris : Librairie de la Société de l’Histoire de France. Date : 1320-24.

Froissart, Chroniques, Premier livre, éd. G.T. Diller, édition du manuscrit de Rome Reg. lat.

869, 1972, Genève. Droz. Date : entre 1369 et 1377 (pour le premier livre).

L'estoire de Griseldis en rimes et par personnages, éd. M. Roques; édité d'après le manuscrit unique de la BN, 1957, Genève. Droz. date : 1395.

\section{E : textes du 15e}

Les 15 Joyes du Mariage, éd. J. Rychner, 1963, Genève : Droz. Date : vers 1400.

Enguerrand de Monstrelet, Chronique (extrait), éd. L. Douët d’Arcq, 1857-1862, Paris. Société de l'Histoire de France (SHF). Date : 1441.

Antoine de la Sale, Jehan de Saintré, éd. J. Misrahi, et C. A. Knudson, 1965, Genève. Droz. Date : 1456.

Le Roman du Comte d'Artois, éd. Charles Seigneuret, 1966, Genève : Droz. Date : 145367.

Les Cent Nouvelles Nouvelles, éd. F.P. Sweetser, 1966, Genève : Droz. Date : 1456-1467.

La Farce de Maistre Pierre Pathelin, éd. J. Dufournet, 1986, Paris : Garnier-Flammarion. Date : 1456-1469

Le Roman de Jehan de Paris, éd. E. Wickersheimer, 1923, Paris : Champion (Librairie ancienne). Date : 1494 
Commynes, Mémoires, éd. J. Calmette, 1964-65, Paris. Belles Lettres (Classiques de l’Histoire de France au Moyen-Age). Date : environ 1490-1505.

\section{$F$ : textes du 16e}

Rabelais, Le Tiers Livre, éd. P. Jourda, 1962, Paris. Garnier. Date : 1546.

\section{Bases de données d'ancien et moyen français :}

Base textuelle Frantext; CNRS - ATILF.

Base de données du Dictionnaire de Moyen Français (DMF) : CNRS-ATILF. Base du français médiéval, ENS-LSH et CNRS (UMR 8503).

\section{Oeuvres citées}

Bloom, Paul; Mary Peterson; Lynn Nadel (éd.). 1996. Language and Space. Cambridge (Mass.) / Londres : MIT Press.

Dirven, René. 1993. Dividing up physical and mental space into conceptual categories by means of English prepositions. In The semantics of prepositions : from mental processing to natural language processing, C. Zelinsky-Wibbelt (éd.), Berlin / New York : Mouton de Gruyter, 73-97.

Gaffiot, Félix. 1934. Dictionnaire Latin-Français. Paris : Hachette.

Godefroy, Frédéric. 1880. Dictionnaire de l'ancienne langue française et de tous ses dialectes du IXe au XVe siècles : composé d'après le dépouillement de tous les plus importants documents manuscrits ou imprimés qui se trouvent dans les grandes bibliothèques de la France et de l'Europe. Paris : H. Champion.

Haspelmath, Martin. 1997. From space to time: temporal adverbials in the world's languages. Berlin : LINCOM EUROPA.

Heine, Bernd; Ulrike Claudi; Friederike Hünnemeyer. 1991. Grammaticalization : a conceptual framework. Chicago : University of Chicago Press.

Jackendoff, Ray. 1990. Semantic structures. Cambridge (Mass.) / Londres : MIT Press.

Kryk-Kastovsky, Barbara. 1996. The linguistic, cognitive and cultural variables of the conceptualization of space. In The construal of space in language and thought, M. Pütz, R. Dirven (éds), Berlin / New York : Mouton de Gruyter, 329-344.

Langacker, Ronald. 1993. Prepositions as grammatical(izing) elements. Leuvense Bijdragen 81, 287-309.

Pütz, Martin; René Dirven (éds). 1996. The construal of space in language and thought. Berlin / New York : Mouton de Gruyter.

Quemada, Bernard (dir). 1990. Trésor de la langue française : dictionnaire de la langue du XIXe et du XXe siècle, 1789-1960. Centre national de la recherche scientifique, Institut national de la langue française. Paris : Gallimard. 
Rey, Alain; J. Rey-Debove. 1987. Le petit Robert. Paris : Dictionnaires Le Robert. Svorou, Soteria. 1994. The grammar of space. Amsterdam : Benjamins.

Talmy, Leonard. 1996. Fictive motion in language and 'ception'. In Language and Space, P. Bloom, M. Peterson, L. Nadel (éds), Cambridge (Mass.) / Londres : MIT Press, 211276.

Talmy, Leonard. 2000. Toward a cognitive semantics. Cambridge (Mass.) : MIT Press.

Tobler, Adolf; Erhard Lommatzsch; Hans Christmann. 1969. Altfranzösisches Wörterbuch. Berlin : Weidmannsche Buchhandlung puis Wiesbaden : F. Steiner.

Traugott, Elizabeth Closs; Ekkehard König. 1991. The semantics-pragmatics of grammaticalization revisited. In Approaches to grammaticalization, E. Traugott, B. Heine (éds), Amsterdam : Benjamins, 189-218.

Vandeloise, Claude. 1986. L'espace en français : sémantiques des prépositions spatiales. Paris : Editions du Seuil.

Von Wartburg, Walther. 1958-1968. Französisches Etymologisches Wörterbuch : eine Darstellung Des Galloromanischen Sprachschatzes. Bâle : Zbinden Druck und Verlag.

Zelinsky-Wibbelt, Cornelia (éd). 1993. The semantics of prepositions : from mental processing to natural language processing. Berlin / New York : Mouton de Gruyter.

Zelinsky-Wibbelt, Cornelia. 1996. How do we mentally localize different types of spatial concepts? In The construal of space in language and thought, M. Pütz, R. Dirven (éds), Berlin / New York : Mouton de Gruyter.

\section{Summary}

The aim of this paper is to investigate a particular aspect of semantic change. Many theories have tried to capture the regularity in semantic change. In this respect, some linguists have claimed that space is the necessary starting point of any semantic development, and others have stated that space is situated at the same distance from the 'semantic core' of a given lexeme as other semantic domains, such as time. I will formulate a partial answer to the question of the primacy of space. Using diachronic corpus data, I will examine prepositions in Old and Middle French (11th to 16th centuries). The prepositions I have focused on are vers, envers, devers, and pardevers, chosen from a wider corpus of prepositions (which I have studied in the same way) because of their semantic behavior, which is particularly complex, and which I try to explain here in detail. 